\title{
FRAGMENTATION OF VERY HIGH ENERGY HEAVY IONS
}

\author{
M. GIORGINI ${ }^{1, a}$ and S. MANZOOR ${ }^{2, a}$ \\ ${ }^{1}$ Dip. di Fisica and INFN, V. le C. Berti Pichat 6/2, I-40127 Bologna, Italy \\ E-mail: miriam.giorgini@bo.infn.it \\ ${ }^{2}$ Radiation Physics Division, PINSTECH, P.O. Nilore, Islamabad, Pakistan, \\ E-mail: shahid.pins@dgcc.org.pk,manzoor@bo.infn.it
}

Presented by M. Giorgini at the Int. Conf. on Structure of the Nucleus at the Dawn of the Century, Bologna (Italy), May 29-June 3, 2000.

\begin{abstract}
A stack of CR39 $\left(\mathrm{C}_{12} \mathrm{H}_{18} \mathrm{O}_{7}\right)_{n}$ nuclear track detectors with a $\mathrm{Cu}$ target was exposed to a $158 \mathrm{~A} \mathrm{GeV}$ lead ion beam at the CERN-SPS, in order to study the fragmentation properties of lead nuclei. Measurements of the total, break-up and pick-up charge-changing cross sections of ultrarelativistic $\mathrm{Pb}$ ions on $\mathrm{Cu}$ and CR39 targets are presented and discussed.
\end{abstract}

\section{Introduction}

We present experimental results on fragmentation charge-changing cross sections of $158 \mathrm{~A} \mathrm{GeV}$ lead ions (charge $Z=82 e$ ) incident on $\mathrm{Cu}$ and CR39 targets. To detect and identify the relativistic ions, the nuclear track detector CR39 was used. When an ion crosses a nuclear track detector foil, it produces damages at the level of molecular bonds, forming the so called "latent track". During the chemical etching of the detector in a basic water solution, etch-pit cones are formed on both sides of the foil. The base area and the height of each cone are functions of the Restricted Energy Loss (REL) of the incident ion and thus of its charge $Z$ 坥.

\section{Experimental procedure}

A stack made of CR39 nuclear track detectors with a $\mathrm{Cu}$ target was exposed in November 1996 at the CERN-SPS to a beam of $158 \mathrm{~A} \mathrm{GeV} \mathrm{Pb}$ ions. The exposure was performed at normal incidence. The total number of lead ions incident on the stack was about $7.8 \times 10^{4}$, distributed in 8 spots. The central density in each spot was around 1500 ions $/ \mathrm{cm}^{2}$.

The stack had the following composition: 12 CR39 sheets $\sim 0.6 \mathrm{~mm}$ thick, a $\mathrm{Cu}$ target $\sim 10 \mathrm{~mm}$ thick; 38 CR39 sheets $\sim 0.6 \mathrm{~mm}$ thick. In the present

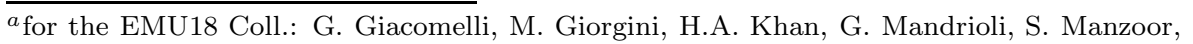
L. Patrizii, V. Popa, I.E. Qureshi, M.A. Rana, M. Sajid, P. Serra, M.I. Shahzad, G. Sher and V. Togo. 


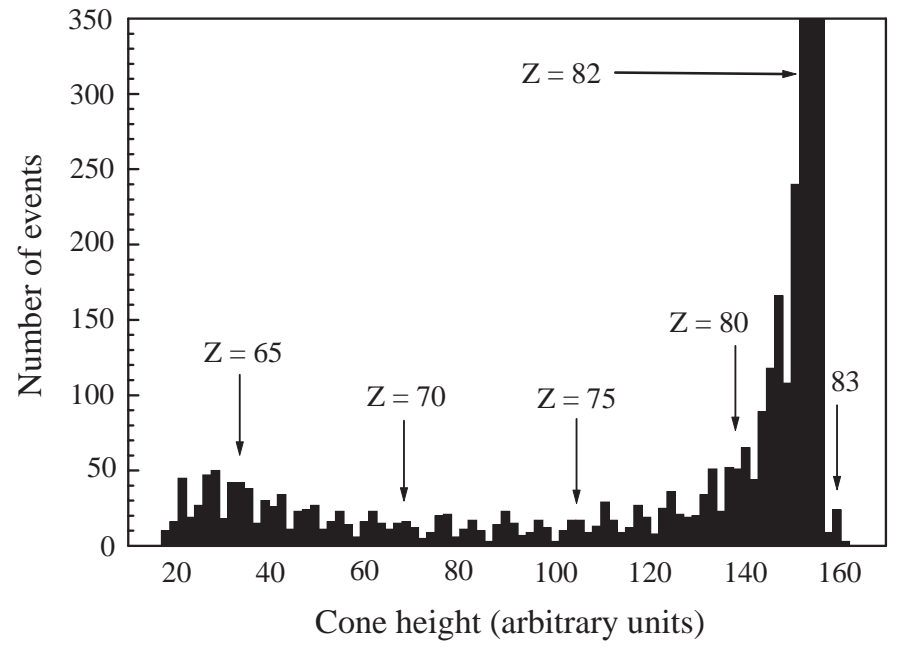

Figure 1: Cone height distribution for $\mathrm{Pb}$ ions and heavy fragments measured on one face of the CR39 sheet immediately after the $\mathrm{Cu}$ target.

analysis, the CR39 sheets immediately before and after the $\mathrm{Cu}$ target and the last sheet of the stack were used. After exposure, the sheets were etched for $72 \mathrm{~h}$ in a $4 \mathrm{~N} \mathrm{KOH}$ water solution at a temperature of $45^{\circ} \mathrm{C}$. Previous calibrations of the detectors have shown that for high $Z$ nuclei, the height of the etched cone is more sensitive to $Z$ than its base area or diameter 3 . In order to separate the lead ions from the nuclear fragments with charge $Z \geq 63 e$, we performed manual measurements of about 6300 cone heights using an optical Zeiss microscope with a magnification of $40 \times$. Fig. 1 1 shows the cone height distribution of $\mathrm{Pb}$ ions and heavy fragments measured on a single face of the CR39 sheet located after the $\mathrm{Cu}$ target. The charge resolution obtained is about $0.2 e$.

\section{Total charge-changing cross sections}

Using the survival fraction of lead ions for the $\mathrm{Cu}$ and CR39 targets, we measured the total charge-changing cross sections of lead ions using the formula:

$$
\sigma_{\text {tot }}=\frac{A_{T}}{\rho_{T} t_{T} N_{A}} \ln \frac{N_{\text {in }}}{N_{\text {out }}}
$$

where $N_{\text {in }}$ and $N_{\text {out }}$ are the numbers of lead ions before and after the target, respectively; $N_{A}$ is Avogadro's number; $\rho_{T}, A_{T}, t_{T}$ are the density, the atomic 
mass and the thickness of the target. The data are indicated by the black points in Fig. 2, the uncertainties are statistical only.

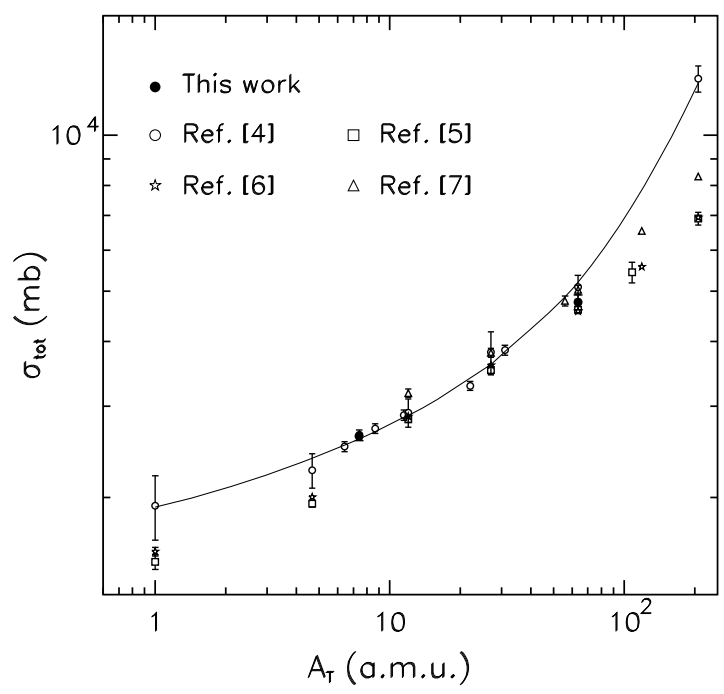

Figure 2: Measured total fragmentation charge-changing cross sections $\sigma_{\text {tot }}$ of $158 \mathrm{~A} \mathrm{GeV} \mathrm{Pb}$ projectiles versus the target mass number $A_{T}$ : the black points are our data on $\mathrm{Cu}$ and CR39, the open points refer to data obtained by a similar experiment ${ }^{4}$ using the same beam. The solid line represents the fit of all the data to formula (2) of ref. [4]. Results from a $10 \mathrm{~A}$ $\mathrm{GeV} \mathrm{Au}$ beam incident on various targets ${ }^{[5-7]}$ are also shown.

As shown in Fig. 2, the data are in agreement with previous data obtained by a similar experiment using the same beam and different targets with atomic masses ranging from 4.7 a.m.u. $\left(\mathrm{CH}_{2}\right)$ to 207 a.m.u. (Pb) The solid line in Fig. 2 is the fit of all the data to formula (2) of ref. [4] which yields $\chi^{2} /$ D.o.F. $=0.7$. Results from other experiments using a $10 \mathrm{~A} \mathrm{GeV} \mathrm{Au} \mathrm{beam}$ incident on various targets ${ }^{[5-7]}$ are also shown in Fig. 2.

\section{Partial fragmentation charge-changing cross sections}

The partial fragmentation charge-changing cross sections of $\mathrm{Pb}$ ions yielding fragments with charge $64 e \leq Z<82 e$ were calculated for the $\mathrm{Cu}$ and CR39 targets using the formulal:

$$
\sigma_{Z}=\frac{A_{T}}{\rho_{T} t_{T} N_{A}} \frac{N_{Z}}{N_{82}}
$$

where $Z=64 e \div 81 e, N_{Z}$ is the number of fragment nuclei with charge $Z$ produced in the target, $N_{82}$ is the number of unfragmented beam nuclei and $\rho_{T}, A_{T}, t_{T}, N_{A}$ have the same meaning as in Eq. (11). In this procedure, the successive fragmentation processes are neglected. The results for the partial fragmentation cross sections of incident lead ions on $\mathrm{Cu}$ and CR39 targets are shown versus $\Delta Z$ in Fig. 3 . 


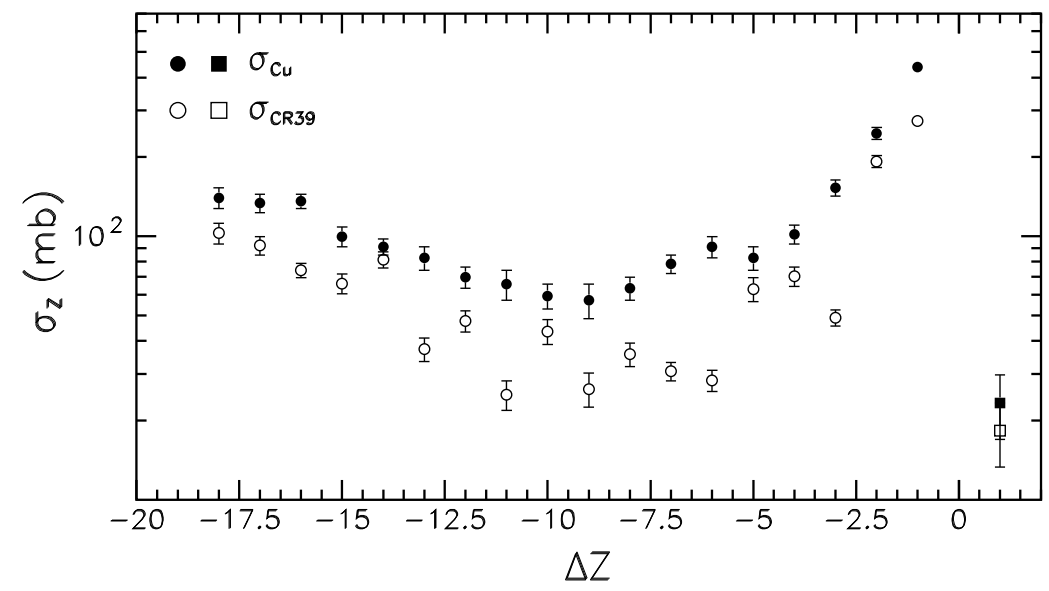

Figure 3: Partial fragmentation charge-changing cross sections for incident lead ions, $\sigma_{Z}$ versus $\Delta Z$ for the $\mathrm{Cu}$ and CR39 targets computed with Eq. (2). The black points refer to $\sigma_{Z}$ for $\mathrm{Cu}$, the open points refer to $\sigma_{Z}$ for CR39. The squares refer to the pick-up cross sections. The errors are only statistical.

The square points in Fig. 3 refer to the charge pick-up cross sections, determined using Eq. (2) where $N_{Z}$ is the number of nuclei with $Z=83 e$ produced in the target.

\section{Acknowledgments}

We thank the CERN SPS staff for the good performance of the exposure, the technical staff of the Bologna INFN and of the PINSTECH Laboratory.

\section{References}

1. R. Fleischer, P.B. Price and R.M. Walker in Nuclear Tracks in Solids, (University of California Press, 1975).

2. S. Cecchini et al., Nuovo Cimento A 109, 1119 (1996).

3. G. Giacomelli et al., Nucl. Instrum. Methods A 411, 41 (1998).

4. H. Dekhissi et al., Nucl. Phys. A 662, 207 (2000).

5. S.E. Hirzebruch et al., Phys. Rev. C 51, 2085 (1995).

6. L.Y. Geer et al., Phys. Rev. C 52, 334 (1995).

7. Y. He and P.B. Price, Z. Phys. A 348, 105 (1994).

8. D.P. Bhattacharyya et al., Nuovo Cimento C 16, 249 (1993). 